\title{
CLUSTER BASED ANALYTICAL METHOD FOR THE LOT DELIVERY FORECAST IN SEMICONDUCTOR FAB WITH WIDE PRODUCT RANGE
}

\author{
Marcin Mosinski \\ Daniel Noack \\ Falk Stefan Pappert \\ Oliver Rose
}

\author{
Wolfgang Scholl \\ Infineon Technologies Dresden \\ Königsbrücker Strasse 180 \\ 01099 Dresden, GERMANY
}

\begin{abstract}
The usual forecast method in semiconductor industry is simulation. Due to the manufacturing environment, the number of processes and the multitude of disturbing factors the development of high-fidelity simulation model is time-consuming and requires a huge amount of high quality basic data. The simulation facilitates a detailed prediction possible, but in many cases this level of detail of the forecast information is not required. In this paper, we present an alternative forecast method. It is considerably faster and the results for a subset of parameters are comparable to simulation. The solution does not need a complete fab model but a limited mathematical system and some fast algorithms which make the forecast of important parameters or characteristics possible. The prediction is based completely on statistics extracted from historical lot data traces. It is already implemented and tested in a real semiconductor fab environment and we also present some validation results.
\end{abstract}

\section{INTRODUCTION}

The manufacturing of semiconductors is one of the most complex industrial production processes. Semiconductor factories contain several hundred machines and products and can be classified as job shop manufacturing environments. The production of each product is described by a processing sequence called route. This sequence includes operations with the assignment of specific machine group. As a consequence of the variety of products, different processes have a specific recipe on individual tools. They describe the kind of process, duration and many other parameters. It is a typical manufacturing characteristic of semiconductor manufacturing that tools within a production line are visited not only once but repeatedly by a product.

The common and feasible forecast method used for this kind of scenario is simulation (Bagchi et al. 2008; Scholl et al. 2010). Based on the manufacturing environment, number of machines and multitude of disturbing factors the creation of simulation model is quite complicated (Kohn et al. 2009). First the simulation expert requires a highly detailed model of several tools with all their deterministic characteristics such as, execution speed, process duration, and capacity. Furthermore the stochastic behavior parameters, such as, availability, failure rate, and period of repairs, are needed. Additionally the model configuration needs further information about tool assignments for all steps in each route. The dedication matrix is based on an enumeration of all combinations of machines, operations, process approvals and product types. The work pieces in semiconductor manufacturing are usually boxes with several single wafers that are referred to as lots. These lots are repeatedly transported between several tools. This requires a detailed model of the transport system. There are several stochastic factors which decrease forecast quality in sem- 
iconductor manufacturing like: manufacturing errors and their associated procedure execution by experts or rework. The consequence of all these influences is a major challenge in modeling which is based on an enormous amount of data (Kohn et al. 2009). This data volume results in time consuming database queries for data collection and furthermore increases simulation durations of experiments. In real environments like factories data quality is a big challenge. Frequent issues are inconsistency, availability, format of some data sources and similar common data consolidation problems. Furthermore handling of a complete model is not easy because every revision and the following validation is very time consuming. The simulation has of course several positive aspects. It allows conclusions about performance factors on machine level as well as on factory level. In many cases however, there is no need for forecast results at this level of detail (Powers, Goldszmidt, and Cohen 2005). Especially in the area of short-term forecasts there is a demand for alternative solutions. They need to work faster but the quality of results obtained from these methods for a subset of parameters should be comparable to those from simulation. In comparison with simulation it is not necessary to model the complete fab. A limited mathematical model and an appropriate algorithm are able to produce a forecast for mandatory parameters or key performance indicators.

\section{PROJECT OBJECTIVES}

In cooperation with our project partner from semiconductor industry we specified project objectives and forecast software requirements. The forecast horizon is limited to 14 days. We focused on the daily delivery predictions and a bottleneck early warning system for several machine groups in the fab. We used a real medium sized logic fab with a wide product range as environment for our approach. The approach should support common characteristics of semiconductor manufacturing like:

- Hold state - Lots are set to this state, if they have a processing problem or they depend on special production steps. Manufacturing of one or several following moves require the attendance of a qualified person. Almost only development products enter this rare state.

- Sampling - Allowing lots to skip measurement operations, which only need to be done on a certain percentage of lots.

- Rework - Remanufacturing after processing or out of spec problems.

\section{CONCEPT}

The Alternative Forecast Method - AFM - uses just one exclusive data source, even to extract detailed historic lot movement information. The work pieces have several characteristics and parameters which need to be stored implicitly. The forecast calculation is based on a statistical evaluation of the duration between start and target operations from historical data. The time interval differs for different manufactured products, therefore AFM uses lot grouping rules based on product characteristics. For an improved accuracy the method needs a second level of classification. On this level a clustering is applied which is based on specific predefined lot attributes like priority or tardiness. For the prediction calculation the method needs the current lot position on the route and its corresponding class. This allows the calculation of the arrival time at the target operation by adding the previously defined duration.

\subsection{Lot Classification}

The key difference of AFM compared to other analytical forecast methods in semiconductor industry is the adoption of a double-stage lot classification in groups called AFM-speed-classes. In the first stage, we classify the lots with help of product affiliation. The lots in the fab move on predetermined routes. Therefore it is possible, that the several products on their way from operation A to operation B are manufactured with a different number of operational steps. A variation of throughput times associated with recipe dependent process durations occur even on the same operation. The second classification stage happens in already identified product groups and is regulated by significant lot properties. To meet the classification 
requirements, it is necessary to find the parameters with the most influence on the cycle time. The factors can be separated into two groups:

- Basic, well-known, thus factory independent - valid for the most semiconductor factories, for example: wafer number or priority

- Particular, thus factory specific - bound to one factory, for example: lot-owner (lot is productive, development or engineering)

We determine the influence of parameters for AFM by means of clustering algorithms using historical trace data. For the well-known factors, we confirmed the validity of our hypothesis about group building. We flag the data points with the same characteristics. Following that we evaluate grouping opportunities. As shown in Figure 1, the cycle time can be divided based on priority. Figure 2 illustrates the clustered values with regard to tardiness. It is a typical example of a factory specific property, because the dispatching rules of the examined fab apply due dates to calculate the lot position in the dispatch list. Using these factors we first cluster the lot cycle times and then assign a parameter to describe the set.

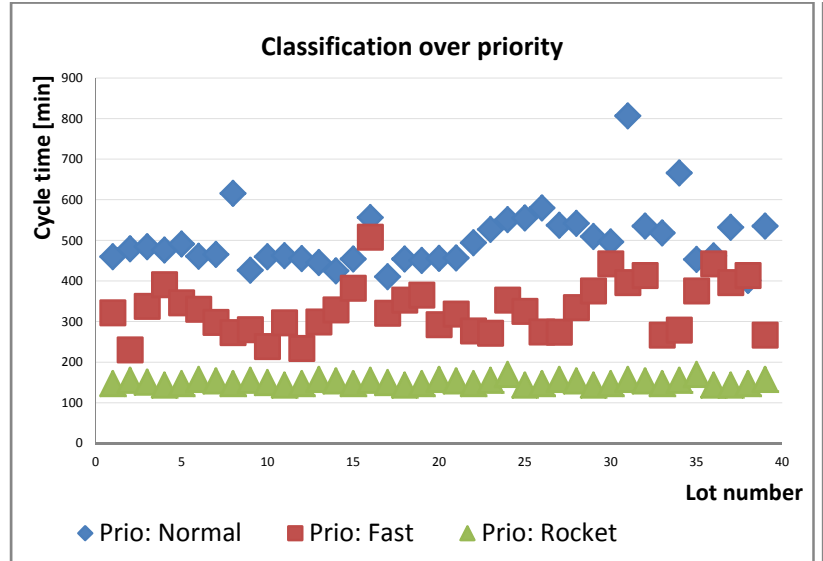

Figure 1: Clustering with the help of priority

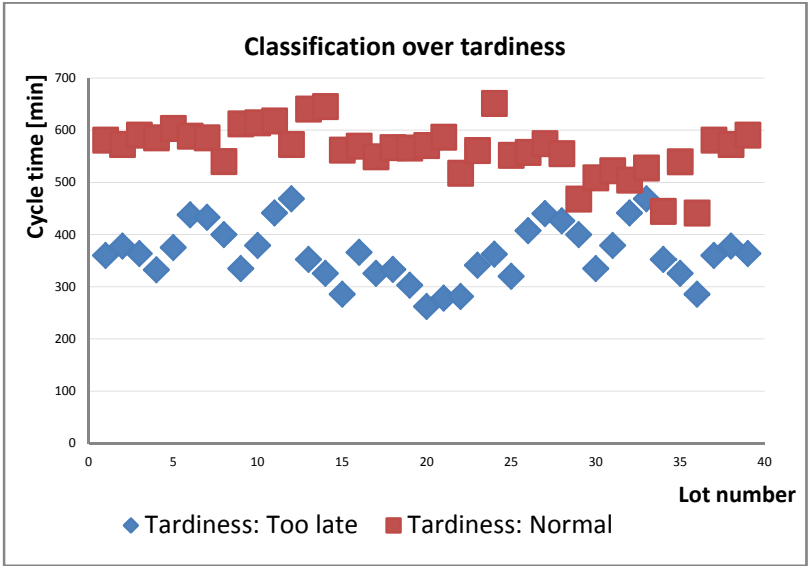

Figure 2: Clustering with the help of tardiness

Of course, it is possible to improve the classification with other parameters, but it is important, that every cluster contains a sufficient number of representatives. Otherwise the calculated average cycle time is not statistically relevant. A too small number of data points in some groups may force us to implement equivalence classes. When the calculation of the cycle time for any group is impossible, the AFM algorithm searches for the value of a corresponding set and multiplies it with a constant factor describing the discrepancy of the time intervals between operations. The factors and equivalence classes are problemspecific and need to be precisely defined with statistical analysis or other experiments.

\subsection{Forecast Elements}

The forecast process is split into four parts: Data collection, statistics generation, forecast calculation and reporting. The data collection component reads the historical information from the manufacturing execution system (MES) and transfers this information in a format compatible with an Oracle database. The analytical algorithms use this data to generate or update the statistics. The statistics modification happens in predefined time periods. The forecast calculation module extracts the current fab state from the MES, as well as the prediction estimates. The reporting algorithms visualize results corresponding to customer requirements and area of application. 


\subsubsection{Data Collection}

In a previous project (Kohn et al. 2009) we used the same data base for simulation and AFM scheduling approaches. After successful completion of the feasibility study, we decided to separate the forecast software completely and make it autonomous. According to our concept we just use the lot trace as single data source. Thus, the separation was not too complicated. The software extracts the required MES data and stores it in a separate database. The calculation of the statistics is independent from the other computations.

\subsubsection{Statistics Creation}

The basic idea of the forecast method is the projection of analytical conditioned information into the future. The data includes the statistics about the time interval from the start operation to the target operation, the sampling, and the hold states. The cycle time and hold time information are affiliated with each other, because both of them have an influence on the prediction of the lot arrival time points. The evaluated statistical cycle time from start operation $A$ to target operation $B$, stat $C T_{A \rightarrow B}$ is calculated as follows:

$$
\begin{gathered}
\operatorname{statCT}_{A \rightarrow B}=\left\{\frac{\sum_{i=0}^{n} W_{i} *\left(\forall x_{i}\left(c t\left(x_{i}\right)-h t\left(x_{i}\right)\right)\right)_{\text {med }}}{\sum_{i=0}^{n} G_{i}}+G_{\text {last }}\left(\text { last_statCT }_{A \rightarrow B}\right)\right\} /\left(1+G_{\text {last }}\right), \\
\text { where } \forall i\left(\operatorname{ct}\left(x_{i}\right)-h t\left(x_{i}\right)>0\right) \neq \emptyset
\end{gathered}
$$

The value $\operatorname{statCT}_{A \rightarrow B}$ describes the current fab state as detailed as possible. We implemented for all data sets the weight $W_{i}$. It negatively correlates with the age of records to reduce the effect of older data, thereby simplifying the handling and calculation. The weights $W_{i}$ for the intervals are fab model specific and should be defined by some analysis. We use in the implemented version for our project partner three of these intervals:

- $\quad$ Last two weeks - with weight $=1$

- $\quad$ Last month - with weight $=0.5$

- $\quad$ Last 3 months - with weight $=0.1$

After assigning values to intervals $i$ and weights $W_{i}$, we start the calculation. First we need the cycle time $c t\left(x_{i}\right)$, to move lot $x_{i}$ from operation $A$ to $B$. From there we subtract the time $h t\left(x_{i}\right)$ the lot was in the hold state between $A$ and $B$. Consequently we calculate processing and waiting times without abnormal breakdowns. This routine will be repeated for every lot $x$. For each interval $i$ we estimate representative values with the help of the median function across all $c t\left(x_{i}\right)-h t\left(x_{i}\right)$. Then we calculate the weighted average over all intervals $i$. Finally we complement the formula with a valid cycle time for the examined route section (last_statCT $T_{A \rightarrow B}$ ) and we store it implicitly with weight $G_{\text {last }}$. This method is working well if for each time interval there is at least one representative lot $x$. It is very important to find out if the route section time for the lot is relevant. We use an outlier filter and make plausibility checks to protect the statistics from corruption.

A lot which is moving on a specified route can pause on every operation, due to special, unexpected processing events. This can have a variety of reasons:

- Special processing supervised by a qualified employee - Mostly short, conditional on waiting for workforce

- Process abortion due to failures or measurements outside of specifications - Duration and probability not easy to define

- Process problem for a product - Common issue in the past

- Product dependent unusual processing - Mostly on development routes, frequently and not common modeling. 
The sum of hold durations in the process chain generates the major part of the noise and complicates cycle time estimation. The hold state statistics collection is different from the cycle time data collection. The information about hold probability and hold length are stored for every operation of each product. First we try to find a large number of data sets with complete information about the lot number, lot hold events including hold duration of an examined operation for a given product. After the recalculation of the lot trace data in a given format the calculation of hold state descriptive parameters is not complicated. The result is the probability of holds for all lots and the representative duration over the median of several, congruent data sets. For this statistic calculation, we need to pay special attention to the correctness of the periodic update to prevent data corruption. All major breakdowns or problems with the complete machine group cause an abnormal accumulation of hold states. So it is very important to develop and use plausibility filters. The AFM implements a three-stage correction algorithm:

- Minimum [5 min] and maximum [2 days] duration

- Plausibility of probability

- Plausibility of duration

The plausibility checks compare the stored data values from the past. It assumes to start with an excellent initialization of the statistics. We need to find a long period of time without an abnormal disturbance. This is almost impossible in a highly loaded semiconductor fab. Therefore the user needs to sanitize the data manually.

The lot delivery forecast for dedicated operations has to respect sampling regulations. The sampling rule defines for every product and process step a relationship between lots which should be processed and lots which skip the operation. This problem occurs mostly at measurement operations. The statistics are calculated similarly to hold probabilities. The sampling rate information is available for a specified product and all target operations. In productive mode, we use constant sampling rates, structured in 6 groups:

- $0 \% \ldots 10 \% \quad$ - All lots skip the measurement operation

- $10 \% \ldots 27 \%$ - Every 4 lots, the lot must be handled

- $27 \% \ldots 40 \%$ - Every 3 lots, the lot must be handled

- $40 \% \ldots 60 \% \quad$ - Every 2 lots, the lot must be handled

- $60 \% \ldots 80 \% \quad-3$ lots out of 4 must be handled

- Over $80 \%$ - All lots must be handled

The aforementioned clusters are customized to the sampling rules of the MES. Changes in the fab control system force the user to revise the forecast specification while the statistics are preserved.

\subsubsection{Forecast Calculation}

The forecast calculation is composed of 6 elements and one separated module which is responsible for the reporting. Figure 3 depicts as starting point the snapshot of the current lot positions. The creation of the snapshot is performed quickly by the MES. Therefore we receive the main information about all lots, such as, current operations, waiting times, process progress, and product types. In addition we identify and store additional lot properties which are used for reporting. In the next stage we search for a possible target step for every lot.

The forecast concept supports numerous user-defined actions for a prediction. Every forecast scenario must be specified before the start of the calculation and should just include key steps or work centers. In theory it is possible to make a prediction for a complete fab. For all practical purposes every update of statistics and even forecast calculation consumes far too much time and resources to meet the project requirements. After identification of start and target operations we can estimate the required cycle time

$c t_{F_{A \rightarrow B}}$. For that purpose we need to classify every lot into the AFM-speed-classes, to ensure that ade- 
quate values are selected from the statistics. At the same time we set values for hold time $h t_{F_{A \rightarrow B}}$ for the step sequence from $A$ to $B$, thus $A \rightarrow B$. The estimation is calculated with the formula:

$$
h t_{F_{A \rightarrow B}}=\sum_{i=1}^{n}\left(\left(\left(P R N_{[0,100]} \leq h p_{i}\right)=>h t_{i}\right) \cup\left(\left(P R N_{[0,100]}>h p_{i}\right)=>0\right)\right)
$$

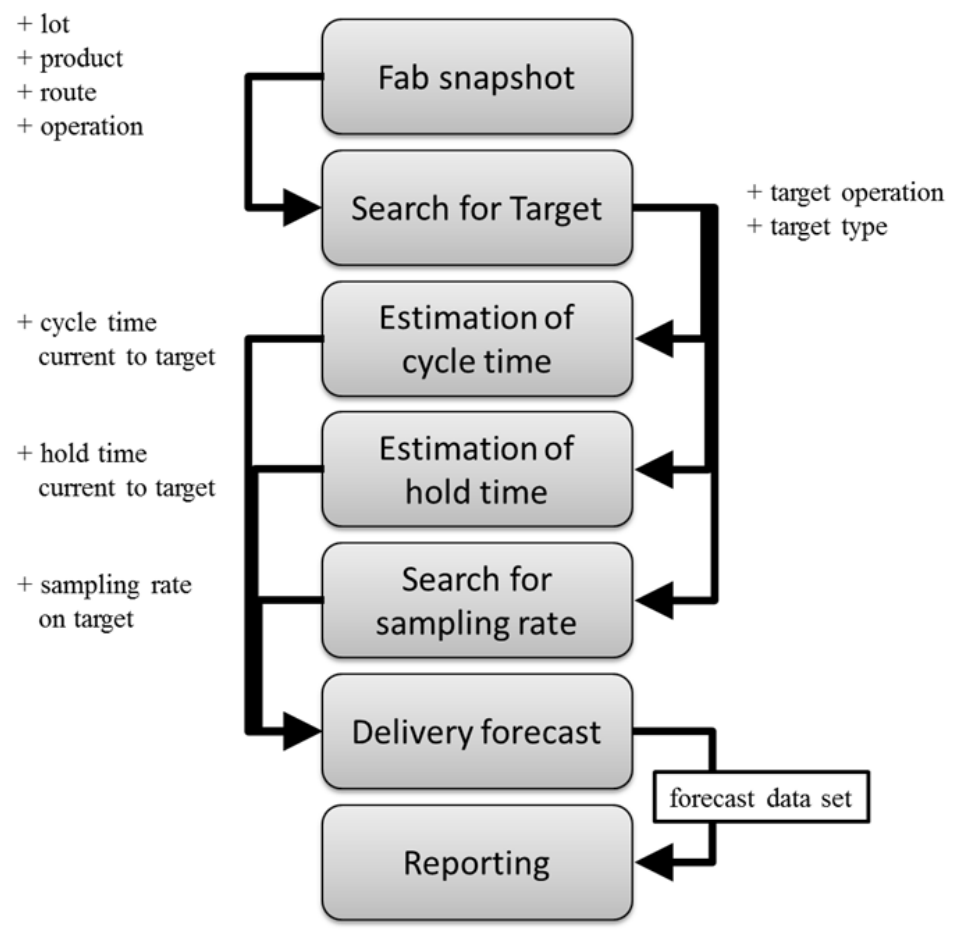

Figure 3: Levels of forecast process

We use uniformly distributed random numbers $P R N_{[0,100] i}$ between 0 and 100 and compare with the hold probability $h p_{i}$. In the first case, if the random number is equal or less than the hold probability $h p_{i}$ on operation $i$, we need to complement the normal process time with statistical hold time $h t_{i}$. In the second case we do not need to add an additional $h t_{i}$. Thus we set it to zero. To calculate the total hold time $h t_{F_{A \rightarrow B}}$ between steps $A$ and $B$ we accumulate the estimated hold time per operation for all operations $n$. The sampling rate of the target operation is then adopted from the sampling statistic for each product.

After we collect or calculate all required data, we can start the forecast. First we identify the arrival time stamp on a target operation for each lot in the fab. These values result from the addition of cycle time $c t_{F_{A \rightarrow B}}$ and hold time $c t_{F_{A \rightarrow B}}$ to the time of release into the current operation $A$ for a certain lot. This is the way the single lot forecast is generated. In the next step we need to convert the single lot forecast into a delivery forecast. The problem is the possibility of lot sampling on some of the target operations. The lots are not marked whether they skip the destination step or not. It makes it necessary to examine the affected lot groupings. The easiest way to explain the principle is with help of an example. Assuming that one of the products has a sampling rate of $25 \%$, which means one lot every four lots will be measured. First we sort the lots by predicted arrival time points. Next we need to define the sampling counter, indicating which lot needs to be processed next. With a simple random function we specify how many lots skip the target and we set the counter. We make a guess which item of our lot list needs to be processed 
first and label consecutively the next significant and legal lots. We just use the lots which are significant for a target operation and match the forecast horizon to build daily delivery sets.

\subsubsection{Reporting}

The last module is just a simple visualization or a recalculation of the delivery data in other ways to forecast. One of them is a bottleneck early warning system on key work centers. After we calculate the delivery forecast, we complement the information with an expected target process time. We can estimate the times from the same data source like other statistics. Once we know the capacity of the reviewed work center, we can estimate its utilization.

\section{IMPLEMENTATION}

The software is composed of three main modules:

- MES data collector

- AFM core

- Reporting und visualization module

The MES data collector is responsible for the communication with the MES Software. It reads, transforms, filters and stores the lot trace and current WIP from the fab. By design, this is not an integrated component of the forecast software in order to be able to adapt quickly to environmental changes.

The main part of the prediction software is the AFM core. The module is completely implemented within an Oracle database. We use Oracle, because it has one of the best opportunities to execute database procedures. We compared the solution with external Java software and analog database based procedures. For the calculation of the sampling statistics an Oracle database takes about a tenth of the time of the Java version. The statistics calculation occurs periodically after reading the trace data from the MES. It involves 5 stages: trace data correction, refresh of forecast target, cycle time statistics update, hold statistics update, and sampling statistics update. It is executed sequentially and takes about 20 hours. The forecast can run in parallel to the update and is fully independent. Therefore it does not need to wait for the statistics update. The prediction needs about 2 minutes for a complete run, 30 seconds for the fab snapshot and 40 seconds for reporting. The estimation of the lot arrivals needs less than 1 minute. Our measurements are designed for a part of products, machines and target steps. We want to support a comparison to other methods. The prediction is based on data from a fab with about 150 products with an average of 250 steps in a route. The target was specified twice per route and there are about 600 lot deliveries per day on all destination operations. The database table size is extremely large for all calculations. For this reason we implemented several advanced cleaning algorithms.

\section{VALIDATION AND RESULTS}

For validation we use about 90 predictions. They were executed daily over a three-month period. The forecast horizon is limited to 14 days. We consider the wafer out of the last operation in every route, i.e., the fab wafer out. This operation at the end of the route has of course no sampling. But during the two weeks before the lot leaves the fab, there are a large number of disturbances like hold that can affect the lot. As a sampling afflicted step we use a typical measurement operation almost in the middle of the route. We separate two kinds of validations:

- Single lot validation - Comparison to the real lot arrival at an operation. The test parameter is the absolute time deviation of the lot arrival between the real and the forecasted values

- Delivery set validation - Comparison of the sum of lots, which arrived per day in the fab and in the forecast. The test parameter is based on the absolute deviation between the real and the forecasted numbers of lots. 
At the beginning we present the validation results of lot arrival times at the target operations. For the comparison we take all predicted delivery information, i.e., every lot at each target. We compare the absolute time deviation. The green curve "AFM - new classification", depicted in Figure 4, represents the validation values with good corresponding AFM-speed-classification to the current fab state.

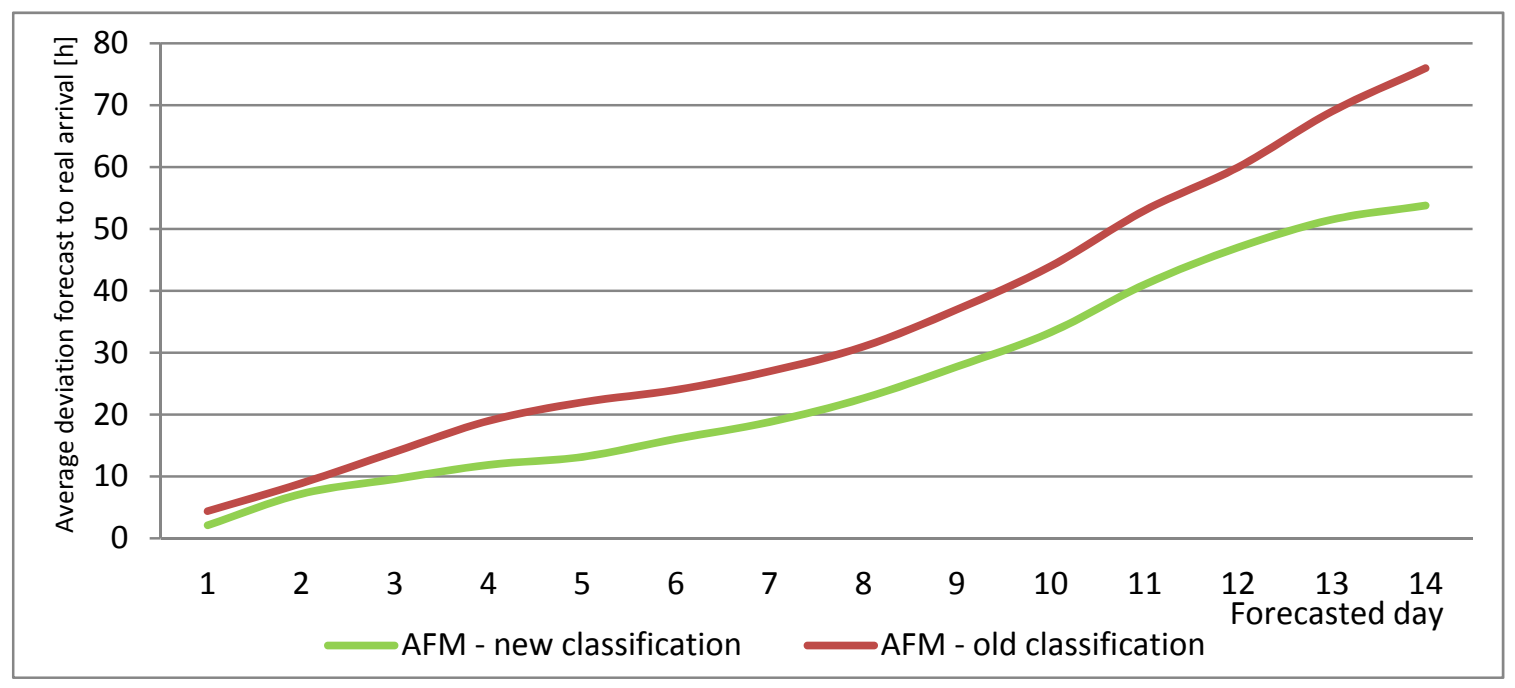

Figure 4: Validation of lot arrival times

The output was created when the factory was highly loaded. The red curve demonstrates the same forecasts with the old classification for the fab capacity utilization of about $60 \%$. In this example we see the importance of the statistics preparation to configure the definition of the AFM-speed-classes accurately. For the first forecast week, the accuracy of the AFM is quite good, because the average time deviation is always below 20 hours. For the second week the deviation values increase considerably faster. On the 12th predicted day we have a discrepancy of almost 2 days in the average lot arrival. This difference makes a single lot forecast invalid. But it is still possible to properly forecast delivery trends.

For the validation of the delivery forecast, we use the real lot arrival stream. We compare the total number of incoming lots to the predefined operation or we use some characteristics to limit the arriving lot streams. Figure 5 shows the probability of matching the real delivery with the help of the forecast. The probability $p b$ is calculated by the formula:

$$
p b=\left\{\begin{array}{l}
Q_{\text {real }}>\text { abs }\left(Q_{\text {real }}-Q_{\text {forecast }}\right) \Rightarrow 100-\text { abs }\left(1-Q_{\text {forecast }} / Q_{\text {real }}\right) * 100 \\
Q_{\text {real }} \leq \operatorname{abs}\left(Q_{\text {real }}-Q_{\text {forecast }}\right) \Rightarrow 0
\end{array}\right.
$$

where $Q_{\text {real }}$ is the real quantity and $Q_{\text {forecast }}$ stands for the forecasted quantity. On this diagram we see that the green curve for the delivery on the main steps without sampling provides better results than the red one. The explanation is the use of further statistics, i.e., the sampling statistics. Apart from the sampling operations, the charts represent completely different operations in the route. Therefore it is not easy to explain the probability differences in this case.

In general we observe over $90 \%$ forecast probability for lot delivery in the first 3 days. In the first predicted week, the average probability is about $88 \%$. For the second week, in the normal case, just a future trend prediction is possible, because the quality of the prediction results deviates between $75 \%$ and $87 \%$. 
Mosinski, Noack, Pappert, Rose, and Scholl

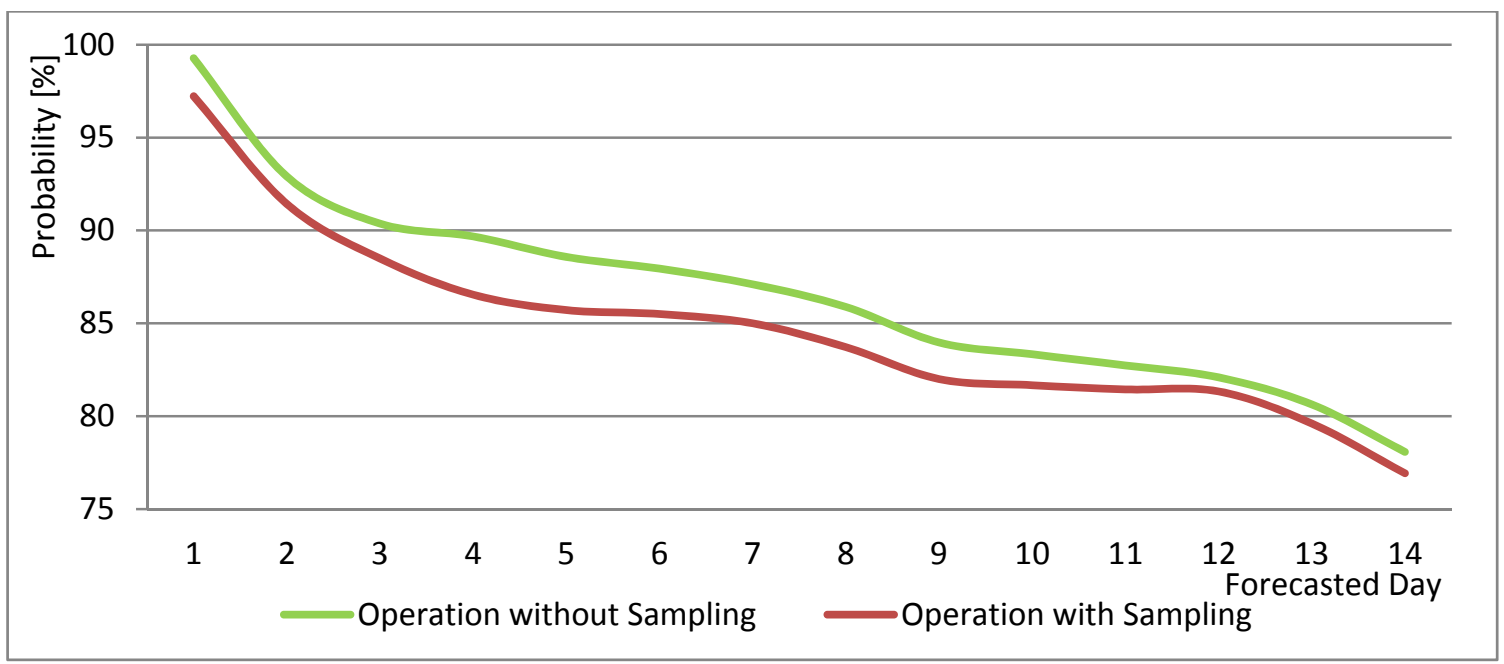

Figure 5: Forecast probability of number of incoming lots

The validation phase exposed strengths and weaknesses of AFM. The advantages are short and stable forecast calculation times, good accuracy in the first predicted days and high accuracy for prioritized lots. We achieve better forecast results for bulk products. Problematic are the products manufactured in small batch series, development products or completely new products. For this kind of products, we usually do not have enough samples to generate statistically significant values. But the forecast generated with our parameters was nevertheless better than the forecast calculated with planning times (Powers, Goldszmidt, and Cohen 2005). The major disadvantage of AFM is the missing reaction to machine downs or bottleneck situations, immediately before target operations. If there is no common incident, then it has no influence on the statistics, and the algorithms works with default data.

\section{CONCLUSIONS}

In this paper we introduce a forecast method that is applicable for the performance prediction in semiconductor manufacturing. We implemented a software prototype, based on the method and tested it in a real manufacturing environment. We demonstrate that the method produces useful forecasts for the provided time period.

The research project shows that it is not straightforward to implement this kind of methods in a real factory. The environment complexity and amount of required data is huge. The software engineering part and implementation is extremely important to create an acceptable forecast. Furthermore the hardware requirements to predict target values in almost real time are critical. The forecast calculation for all products and for all operations in a route is nearly impossible within the targeted time frame. The method is very useful for several fab applications. The biggest advantage is the small maintenance effort after implementation. The reason for this is the minimal required amount of basic data to solve the described problem.

The next steps involve extending the AFM approach with self-validation modules. The algorithm is still based on the same data source. It checks and compares every forecast data set with the reality in realtime. The intention is to further include the changes of equipment availability to improve the forecast. Further improvement of the AFM can be achieved by introducing additional data sources which provide data about current tool conditions as well as failure and maintenance statistics. In an additional future validation part we want to compare detailed results from the AFM method with a complex online simulation solution. 


\section{REFERENCES}

Bagchi, S., C.-H. Chen-Ritzo, S. T. Shikalgar, and M. Toner. 2008. "A Full-Factory Simulator as a Daily Decision-Support Tool for 300mm Wafer Fabrication Productivity." In Proceedings of the 2008 Winter Simulation Conference, edited by S. J. Mason, R. R. Hill, L. Mönch, O. Rose, T. Jefferson, and J. W. Fowler, 2021-2029. Piscataway, New Jersey: Institute of Electrical and Electronics Engineers, Inc.

Kohn, R., D. Noack, M. Mosinski, Z. Zhou, and O. Rose. 2009. "Evaluation of Modeling, Simulation and Optimization Approaches for Work Flow Management in Semiconductor Manufacturing." In Proceedings of the 2009 Winter Simulation Conference, edited by M. D. Rossetti, R. R. Hill, B. Johansson, A. Dunkin, and R. G. Ingalls, 1592-1600. Piscataway, New Jersey: Institute of Electrical and Electronics Engineers, Inc.

Powers, R., M. Goldszmidt, and I. Cohen. 2005. "Short Term Performance Forecasting In Enterprise Systems." In Proceedings of the 11th ACM SIGKDD International Conference on Knowledge Discovery in Data Mining, 801-807. New York, NY, USA: ACM.

Scholl, W., B. P. Gan, D. Noack, P. Preuss, M. L. Peh, P. Lendermann, and O. Rose. 2010. "Towards Realization of a High-Fidelity Simulation Model for Short-term Horizon Forecasting In Wafer Fabrication Facilities." In Proceedings of the 2010 Winter Simulation Conference, edited by B. Johansson, S. Jain, J. Montoya-Torres, J. Hugan, and E. Yücesan, 2563-2574. Piscataway, New Jersey: Institute of Electrical and Electronics Engineers, Inc.

\section{AUTHOR BIOGRAPHIES}

MARCIN MOSINSKI is a PhD student at Dresden University of Technology. He is a member of the scientific staff of Professor Oliver Rose at the Chair of Modeling and Simulation. He received his M.S. degree in computer science from Dresden University of Technology. His research interests include forecasting of complex problems in manufacturing facilities and the statistical data analysis. His email address is marcin.mosinski@infineon.com.

DANIEL NOACK is a Research Engineer at D-SIMLAB Technologies. His focus is on simulation and simulation-based optimization. He is also a PhD student at Dresden University of Technology. He is a member of the scientific staff of Professor Oliver Rose at the Chair of Modeling and Simulation. He received his M.S. degree in Computer Science from Dresden University of Technology. His email address is daniel@d-simlab.com.

FALK STEFAN PAPPERT is a Research Engineer at D-SIMLAB Technologies. His focus is on simulation-based scheduling and optimization of production systems. Furthermore he is a $\mathrm{PhD}$ student at Dresden University of Technology as a member of the scientific staff of Prof. Dr. Oliver Rose at the Chair of Modeling and Simulation. He has received his M.S. degree in Computer Science from Dresden University of Technology. He is a member of GI. His e-mail address is falk.pappert@mailbox.tu-dresden.de.

OLIVER ROSE holds the Chair for Modeling and Simulation at the Institute of Applied Computer Science of the Dresden University of Technology, Germany. He received an M.S. degree in Applied Mathematics and a Ph.D. degree in Computer Science from Würzburg University, Germany. His research focuses on the operational modeling, analysis and material flow control of complex manufacturing facilities, in particular, semiconductor factories. He is a member of IEEE, INFORMS Simulation Society, ASIM, and GI. His web address is www.simulation-dresden.com and his email address is oliver.rose@tudresden.de. 
WOLFGANG SCHOLL works as a Senior Staff Expert for modeling and simulation for Infineon Technologies in Dresden (Germany). He studied Physics at the Technical University of Chemnitz (Germany) and graduated in solid-state physics in 1984. From 1984 to 1995 he worked as a process engineer for ZMD in Dresden. In 1996 he joined Infineon Technologies and initially worked on the field of capacity planning. Since 2003 he is responsible for fab simulation. His email address is wolfgang.scholl@infineon.com. 